\title{
Probing Atomic Scale Dynamics with STEM
}

\author{
Timothy J. Pennycook ${ }^{1,2}$, Lewys Jones ${ }^{1}$, Henrik Pettersson ${ }^{3}$, Valeria Nicolosi ${ }^{3}$ and Peter D. Nellist ${ }^{1,2}$ \\ ${ }^{1 .}$ Department of Materials, University of Oxford, Parks Road, Oxford OX1 3PH, UK \\ 2. SuperSTEM Laboratory, Daresbury, WA4 4AD, UK \\ 3. Trinity College Dublin, College Green, Dublin 2, IR.
}

\begin{abstract}
Aberration correction has enabled atomic resolution at low accelerating voltages in scanning transmission electron microscopy (STEM). The continuing improvement of the resolution of STEM instruments not only provides clearer images, but also enables atomic scale information to be recorded more rapidly. Rapid scanning provides numerous benefits. With a single rapidly scanned image it is often possible to outrun damage in beam sensitive materials. Recording a series of rapidly scanned images provides a means of removing motion blur. Instead of recording a single image with a long dwell time, one can drift correct a series of rapidly scanned images and then average them for a sharper image. Such series can however also be used to record dynamic processes in action. The dynamics could be triggered with a change in temperature, however using specially designed heating or cryogenic sample holders can compromise the stability of the microscope and reduce resolution. An alternative is to use the electron beam itself to provide the energy needed to overcome the energy barriers involved in dynamic processes. If the beam is gentle enough and scanned sufficiently rapidly, useful information about dynamic processes can be revealed.
\end{abstract}

Dynamic STEM imaging has previously provided insights into the origin of white light emission from individual ultrasmall CdSe nanoclusters [1]. It was found that larger monochromatic emitting nanocrystals have a fluctional surface layer up to a nanometer thick surrounding their crystalline cores. Nanoclusters smaller than about $2 \mathrm{~nm}$ were found to be completely consumed by the fluctional surface layer. They posses no crystal core and continuously fluctuate under the electron beam. These observations suggested that the ultraviolet light used to stimulate the nanoparticles could also be inducing dynamic fluctuations. Finite temperature density functional theory (DFT) simulations confirmed that the energy imparted by the photons causes dynamic fluctuations and revealed that the structural changes caused the band gap to fluctuate resulting in a white time averaged emission spectrum.

More recently we have used dynamic STEM to study a solid state chemical reaction. Starting with a thin flake of $\mathrm{Mn}_{3} \mathrm{O}_{4}$ ' we first nucleated a region of $\mathrm{MnO}$ by exposing a small section along the edge of the flake to the electron beam for a prolonged period of time. Electron energy loss spectroscopy (EELS) confirms the identities of the two materials. The beam was then rapidly scanned over the region,

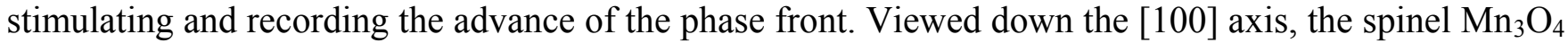
has three types of atomic columns as indicated in the high angle annular dark field (HAADF) image shown in Figure 1. Columns types A and B both contain octahedrally coordinated Mn 3+ atoms, but the $\mathrm{B}$ type columns contain half as many $\mathrm{Mn}$ atoms as type A columns and hence appear darker. Type C columns contain tetrahedrally coordinated Mn 2+ atoms, and also contain half as many Mn atoms as type A columns and so appear similarly intense to type B columns.

When the spinel is viewed down the [100] direction, the MnO appears in the [110] orientation. Playing the time series of images as a movie one sees the adjacent pairs of type $\mathrm{C}$ columns at the phase front 
fluctuating back and forth as if uncertain to remain separate or form a single column. Meanwhile the type B sites at the phase front gradually become brighter, indicating that they are filling up with additional Mn atoms. Once the type B sites have reached the same intensity as the neighboring type A sites, the pairs of type $\mathrm{C}$ columns finally coalesce into single columns. These new columns also have the same intensity as the neighboring type A columns. DFT calculations confirm that these two steps convert the spinel $\mathrm{Mn}_{3} \mathrm{O}_{4}$ into $\mathrm{MnO}$, and the phase front continues its advance by repeating them in the next planes. The dynamic imaging also reveals the likely source of the Mn atoms needed to fill in the vacant sites in the spinel structure. Mn atoms can be seen diffusing along the edge of the material, and they are likely able to diffuse over the other surfaces of the flake. The electron beam is known to eject $\mathrm{O}$ atoms in manganese oxides, so it is likely free $\mathrm{Mn}$ atoms are created on the surfaces and diffuse to the phase front. The results illustrate the advantages of applying STEM to study dynamic processes. Zcontrast imaging offers interpretability and the ability to quantify changes in occupancy while EELS offers additional robust compositional and chemical information [2].

\section{References:}

[1] T. J. Pennycook et al, Nanoletters 12 (2012) p. 3038.

[2] This research was supported by the EPSRC through the UK National Facility for AberrationCorrected STEM (SuperSTEM) and the ERC starting grant 2DNanoCaps (VN and HP).

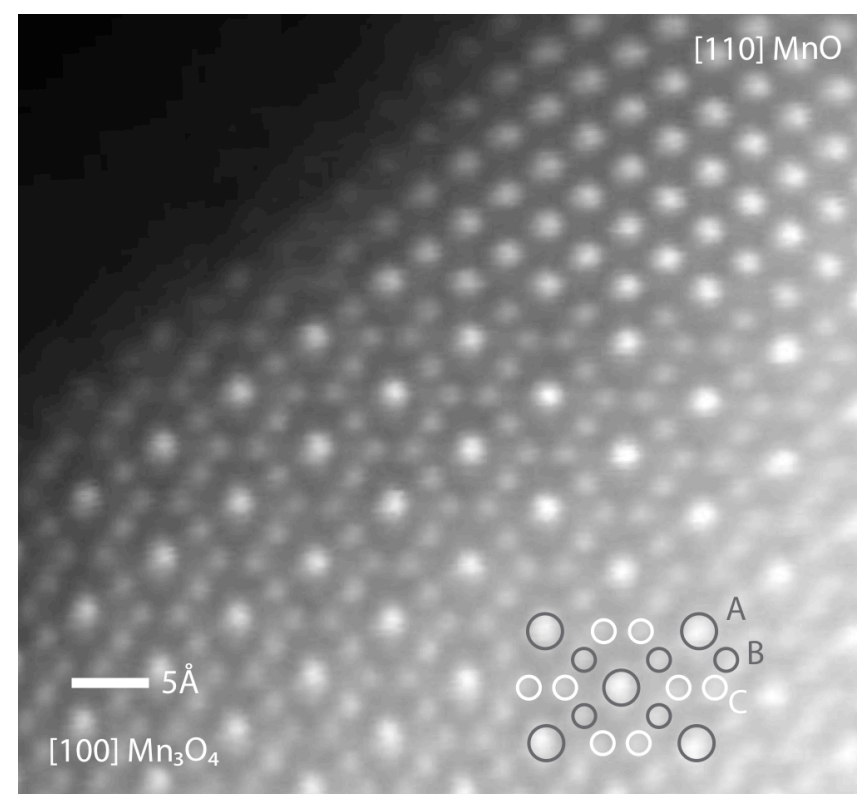

Figure 1. HAADF image of phase front between $\mathrm{MnO}$ (top) and spinel $\mathrm{Mn}_{3} \mathrm{O}_{4}$ (bottom).
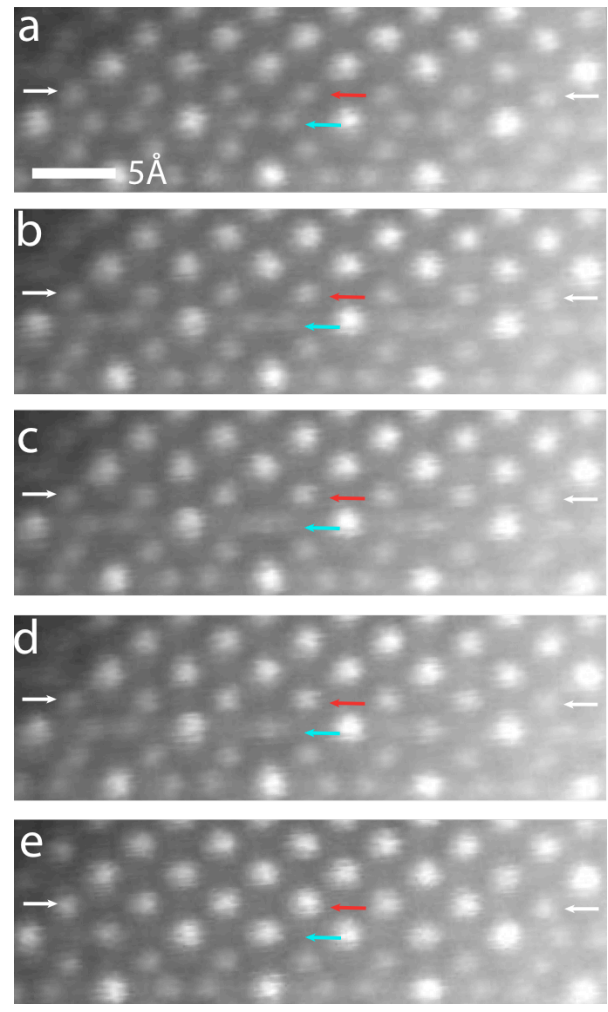

Figure 2. Sequential series of images each created from four frames of a HAADF movie of the phase change. The white arrows indicate the initial position of the first monolayer of the spinel next to the phase front. The red and blue arrows point to what are initially a B type column and a pair of $C$ type columns respectively. 